\title{
Morfometría, Planimetría y Estereología en el Hueso Sesamoídeo Distal en Manos de Equinos con y sin Síndrome Navicular
}

\author{
Morphometry, Planimetry and Stereology in the Distal Sesamoid Bone \\ in Equine Foot with and without Navicular Syndrome
}

Paulo Salinas",;*; Saúl Figueroa*; Carlos Carrasco* \& Rodrigo Bañados****

SALINAS, P.; FIGUEROA, S.; CARRASCO, C. \& BAÑADOS, R. Morfometría, planimetría y estereología en el hueso sesamoídeo distal en manos de equinos con y sin Síndrome Navicular. Int. J. Morphol., 32(1):357-363, 2014.

RESUMEN. El Síndrome Navicular (SN) es una patología claudicógena bilateral crónica, degenerativa y progresiva, que compromete al hueso sesamoídeo distal (HSD), la bolsa podotroclear, ligamentos y a las superficies adyacentes al tendón del músculo flexor digital profundo en manos de equinos. La hipótesis de que las características morfológicas del HSD en la mano de equinos varían en aquellos que presentan SN fue evaluada en este estudio. Para determinar cambios morfológicos en el HSD en manos de equinos con SN se realizó un estudio morfométrico, planimétrico y estereológico. Fueron evaluados dos grupos: No Afectadas (n=11) y Síndrome Navicular $(\mathrm{n}=11)$. Se evaluaron variables tales como peso, volumen, mediciones lineales, área y parámetros estereológicos tales como densidad de número $\left(\mathrm{N}_{\mathrm{A}}\right)$, volumen $\left(\mathrm{V}_{\mathrm{V}}\right)$ y superficie $\left(\mathrm{S}_{\mathrm{V}}\right)$. Los resultados obtenidos indican que a pesar de existir un proceso patológico e inflamatorio en la región del HSD que induce cambios mesoscópicos y microscópicos atribuibles a SN, no lograrían modificar sus características macroscópicas.

PALABRAS CLAVE: Equino; Síndrome navicular; Hueso sesamoídeo distal; Morfometría; Planimetría; Estereología.

\section{INTRODUCCIÓN}

El hueso sesamoídeo distal (HSD) es un componente de la articulación interfalángica distal incluído en la úngula, ubicado entre la falange media y distal en la cara palmar de la mano y pié del equino. Se describe una cara articular y flexora, además un margen proximal y distal. A pesar de su pequeño tamaño, desempeña un rol importante en la estabilización de la articulación interfalángica distal y en el movimiento de los miembros. Proporciona un ángulo de inserción ligamentosa que ejerce el rol de "polea" para el tendón del músculo flexor digital profundo el cual ejerce una fuerza compresiva sobre la falange media y distal. Alteraciones en el HSD en equinos de deporte han demostrado que las mayores fuerzas compresivas se aplican en la fase de propulsión del miembro torácico durante la marcha (Bowker et al., 2001). Esto ocurre durante la extensión de la articulación interfalángica distal, con un incremento en la presión que ejerce el tendón del músculo flexor digital profundo sobre la cara flexora, aumentando el contacto y tensión entre la falange media y distal, ligamentos sesamoídeos colaterales y el HSD (Dyson et al., 2011). Los procesos patológicos que afectan al HSD tienen probablemente diferentes etiopatogénesis. Si bien existe una fuerte evidencia de que las fuerzas biomecánicas pueden resultar en el fracaso de la adaptación funcional y modelado excesivo, lo más probable es que cada tipo de enfermedad o lesión tiene una causa multifactorial.

El Síndrome Navicular (SN) se define como una patología claudicógena bilateral, crónica, degenerativa y progresiva, que compromete al HSD, la bolsa podotroclear, ligamentos y las superficies adyacentes al tendón del músculo flexor digital profundo (Voute, 2008). Es considerada como la causa de al menos un tercio de las cojeras crónicas en equinos (Dyson et al.). Afecta principalmente al miembro torácico en la región distal de la mano, aunque también se han reportado casos en el miembro pélvico (Wilson et al., 2001). Numerosas razones condicionan a los equinos a ser propensos a esta dolencia, generalmente las lesiones

\footnotetext{
* Laboratorio de Anatomía Veterinaria, Escuela de Medicina Veterinaria, Sede Temuco, Facultad de Recursos Naturales y Medicina Veterinaria, Universidad Santo Tomás, Chile.

** Programa de Doctorado en Ciencias Morfológicas, Facultad de Medicina, Universidad de La Frontera, Temuco, Chile.

**** Servicio de Veterinaria, Carabineros de Chile, Región de La Araucanía, Chile.
} 
musculoesqueléticas son causadas por sobresfuerzos debido al gran peso que deben soportar (Dyson et al.). Por lo tanto, durante la inspección veterinaria que acompaña a la compra de un equino la evaluación del estado del HSD se ha convertido en un factor relevante a considerar durante la realización del examen clínico general. Las diferentes líneas de investigación convergen sus esfuerzos en entender la etiopatogenia, la biomecánica y la búsqueda de mejoras en la terapéutica, sin embargo, antecedentes morfológicos cuantitativos no han sido descritos en el HSD de manos con SN. El presente estudio busca aportar antecedentes cuantitativos respecto a los cambios morfológicos asociados a SN.

\section{MATERIAL Y MÉTODO}

Material biológico. Se utilizaron manos izquierdas de 22 equinos (Equus ferus caballus) destinados a sacrificio por razones no relacionadas con esta investigación (4-10 años; peso promedio: $350 \mathrm{~kg}$ ), que presentaron características externas sugerentes de SN tales como: baja angulación del dedo, paredes largas y concavidad en la cara solear de la úngula y manos sin despalmes correctivos (Stashak, 2004). Fueron sometidas a procedimientos de desangrado, flushing de vasos sanguíneos, depilación, desinfección y rotulado. Posteriormente fueron perfundidas con formalina $10 \%$ tamponada (PBS 4\%) previo a la etapa de diagnóstico.

Diagnóstico de Síndrome Navicular y grupos. Se realizó mediante una evaluación morfológica y radiográfica de las manos que incluyó al HSD, ligamentos y cavidad articu- lar de la articulación interfalángica distal. Se utilizaron dos vistas radiográficas: latero-medial y palmaroproximalpalmarodistal oblicua en $45^{\circ}$ (Kinast, 2001). Los hallazgos radiográficos y criterios utilizados para diagnosticar $\mathrm{SN}$ se describen en Tabla I y II (Floyd \& Mansmann, 2009). Se conformaron los siguientes grupos: No Afectadas (n=11) y Síndrome Navicular $(\mathrm{n}=11)$. Se consideraron manos No Afectadas, aquellas que evidenciaron $\leq 2$ hallazgos con graduación $\leq$ a 2 descritos en Tabla I y $\leq$ a 2 hallazgos descritos en Tabla II, y manos con Síndrome Navicular a aquellas que evidenciaron $\geq 3$ hallazgos con graduación $\geq 3$ descritos en Tabla I $y \geq 3$ hallazgos descritos en Tabla II. Posterior a la etapa de diagnóstico los HSD fueron obtenidos por simple disección.

Estudios. Se realizó un estudio morfométrico, planimétrico y estereológico en el HSD. En el primero, se evaluó medidas lineales ( $\mathrm{mm}$ ) con un pié de metro (Truperß) (Fig. 1), y además se determinó el peso $(\mathrm{g})$ y volumen $(\mathrm{ml})$ mediante una balanza digital y el modelo de determinación volumétrica de órganos propuesto por Scherle (Mandarim de Lacerda, 2003), respectivamente. Se efectuó un corte transversal a nivel de la cresta de la cara flexora seccionando el HSD en dos mitades iguales, las cuales fueron utilizadas en el estudio planimétrico y estereológico respectivamente. En el estudio planimétrico, el área $\left(\mu \mathrm{m}^{2}\right)$ de i) cartílago hialino, ii) fibrocartílago, iii) tejido óseo trabecular y iv) tejido óseo compacto del HSD se determinó mediante el análisis de imágenes digitales de secciones transversales discriminando entre las superficies de los componentes óseos y cartilaginosos, montando sobre éstas un sistema planimétrico de puntos (Sistema test) de distancia y área conocida (Fig.

Tabla I. Hallazgos radiográficos evaluados en hueso sesamoídeo distal.

\begin{tabular}{lccccr}
\hline \multirow{2}{*}{\multicolumn{1}{c}{ Condición }} & \multicolumn{5}{c}{ Grados } \\
\cline { 2 - 6 } & Excelente & Buena & Regular & Pobre & Mal \\
\hline Definición córtico medular & 0 & 1 & 2 & 3 & 4 \\
Esclerosis medular & 0 & 1 & 2 & 3 & 4 \\
Zonas radiolúcidas (quísticas) & 0 & 1 & 2 & 3 & 4 \\
Alteración de cresta (cara flexora) & 0 & 1 & 2 & 3 & 4 \\
Elongación de corteza flexora & 0 & 1 & 2 & 3 & 4 \\
\hline
\end{tabular}

Tabla II. Hallazgos radiográficos evaluados en hueso sesamoídeo distal.

\begin{tabular}{llc}
\hline & \multicolumn{2}{c}{ Presentación } \\
\hline Fragmentos en margen distal & Ausente & Presente \\
Engrosamiento de corteza flex ora & Ausente & Presente \\
Adelgazamiento de corteza flexora & Ausente & Presente \\
Osteofitos o enteseofitos en margen proximal & Ausente & Presente \\
Neoformación ósea en la cara flexora & Ausente & Presente \\
Mineralización distrófica del ligamento sesamoídeo colateral & Ausente & Presente \\
Cavidad articular & Ausente & Presente \\
Asimetría en el hueso sesamoídeo distal & Ausente & Presente \\
\hline
\end{tabular}


2). Para el estudio estereológico, se realizó cortes seriados de $4 \mu \mathrm{m}$ de grosor los que fueron teñidos con HE. Se analizó 5 campos por cada sección histológica. La observación se realizó en un microscopio Axioskop 40 Carl Zeiss ${ }^{\circledR}$ con aumento 1000X mediante el software AxioVision ${ }^{\circledR}$ LE 4.6. Los parámetros estereológicos medidos fueron: densidad de volumen $\left(\mathrm{V}_{\mathrm{v}}\right)$ y densidad de superficie $\left(\mathrm{S}_{\mathrm{v}}\right)$ de osteocitos en tejido óseo compacto y trabecular. Además se determinó el número de osteocitos en tejido óseo compacto y trabecular por $\mathrm{mm}^{2}\left(\mathrm{~N}_{\mathrm{A}}\right)$ mediante planimetría sobre un área calibrada (a) de 19912,34 $\mathrm{mm}^{2}$. Todas las evaluaciones estereológicas consideraron el factor de compresión (Dorph-Petersen et al., 2001) y se realizaron según lo descrito por Mandarim de Lacerda con el test multipropósito M36 y el software Stepanizer® (Tschanz et al., 2011).

Análisis estadístico. El estudio fue de tipo descriptivo, transversal e inferencial. Los datos cuantitativos tales como peso, volumen, longitud, área y densidades se ordenaron en tablas Excel (Microsoft ${ }^{\circ}$ Office $\left.囚 2011\right)$ y se expresaron como promedio $(\mathrm{X}) \pm$ desviación estándar (DE). Para detectar normalidad en los datos se aplicó la prueba D'Agostino-Person. Para observar diferencias estadísticas significativas entre grupos se utilizó la prueba paramétrica de Student o su contraparte

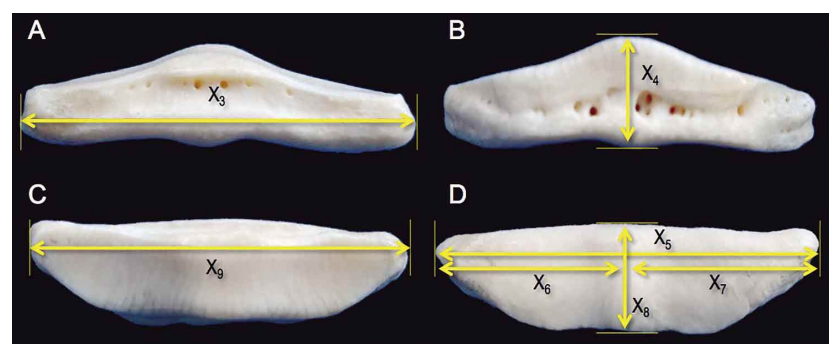

Fig. 1. Hueso sesamoídeo distal de equino. A: margen proximal, $\mathrm{X}^{3}$ : largo mayor. B: margen distal, $\mathrm{X}^{4}$ : grosor. C: cara articular, $\mathrm{X}^{9}$ : largo mayor. D: cara flexora, $\mathrm{X}^{5}$ : largo mayor; $\mathrm{X}^{6}$ : ancho medial; $\mathrm{X}^{7}$ : ancho lateral; $\mathrm{X}^{8}$ : altura mayor. no paramétrica de U Mann-Whitney. Los datos fueron transformados logarítmicamente cuando ambos grupos no coincidieron respecto a demostrar una misma distribución. Se utilizó el Test de Correlación de Pearson (r) para definir la relación entre variables como peso y volumen respecto a la proporción de área de los diferentes tejidos que constituyen el HSD. La significancia estadística para todas las pruebas se estableció para un $\mathrm{p}<0,05$. El análisis de los datos se realizó con el software Prisma ${ }^{\circledR}$ 6.0 para MAC OSX (Graph Pad Software, San Diego CA).

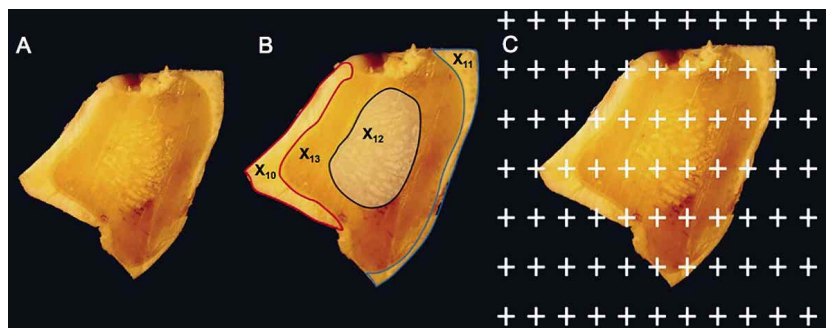

Fig. 2. Hueso sesamoídeo distal en equino. A: corte transversal a nivel de la cresta. B: demuestra la proporción de los cuatro tejidos que componen el HSD, $\mathrm{X}^{10}$ : cartílago hialino, $\mathrm{X}^{11}$ : fibrocartílago, $\mathrm{X}^{12}$ : tejido óseo trabecular, $\mathrm{X}^{13}$ : tejido óseo compacto. $\mathrm{C}$ : ejemplo de test de puntos, donde el punto que intersecta la estructura de interés es contabilizado.

\section{RESULTADOS}

La Tabla III muestra valores promedio de peso, volumen y mediciones lineales en el HSD entre el grupo No Afectadas y Síndrome Navicular. El análisis estadístico no detectó diferencias significativas entre grupos. La Tabla IV muestra el área de los tejidos que componen el hueso sesamoídeo distal observada en los grupos estudiados y la proporción de éstos respecto a la sección transversal (Fig. 3). El análisis estadístico detectó diferencias significiativas entre grupos en

Tabla III. Promedios de peso, volumen y mediciones lineales en el hueso sesamoídeo distal.

\begin{tabular}{lccc}
\hline \multirow{2}{*}{\multicolumn{1}{c}{ Variable }} & \multicolumn{2}{c}{ Promedio $\mathbf{D E}$} & \multirow{2}{c}{$\boldsymbol{p}$} \\
\cline { 2 - 3 } & No Afectadas & $\begin{array}{c}\text { Síndrome } \\
\text { Navicular }\end{array}$ & \\
\hline Peso (g) & $11,08 \pm 0,664$ & $13,58 \pm 2,478$ & 0,1914 \\
Volumen (ml) & $7,750 \pm 0,583$ & $9,583 \pm 1,635$ & 0,0139 \\
Largo may or - margen proximal (mm) & $54,02 \pm 3,240$ & $56,02 \pm 3,435$ & 0,3694 \\
Grosor - margen distal (mm) & $14,46 \pm 0,698$ & $15,11 \pm 2,108$ & 0,4919 \\
Largo mayor - cara flexora (mm) & $51,86 \pm 3,489$ & $53,22 \pm 3,304$ & 0,5044 \\
Ancho medial - cara flexora (mm) & $23,80 \pm 2,824$ & $25,12 \pm 1,918$ & 0,3653 \\
Ancho lateral - cara flexora (mm) & $24,81 \pm 3,562$ & $27,19 \pm 2,364$ & 0,2023 \\
Altura mayor - cara flexora (mm) & $19,65 \pm 1,628$ & $20,49 \pm 1,923$ & 0,4350 \\
Largo mayor - cara articular (mm) & $52,35 \pm 2,908$ & $53,67 \pm 2,685$ & 0,4342 \\
\hline
\end{tabular}


Tabla IV. Área de los tejidos que componen el hueso sesamoídeo distal.

\begin{tabular}{lccc}
\hline \multirow{2}{*}{\multicolumn{1}{c}{ Variable }} & \multicolumn{2}{c}{ Promedio \pm DE $(\boldsymbol{\mu m} 2)$} & \multirow{2}{*}{$\boldsymbol{p}$} \\
\cline { 2 - 3 } & No Afectadas & Síndrome Navicular & \\
\hline Cartílago hialino & $2,03 \times 107 \pm 1,85 \times 106$ & $2,64 \times 107 \pm 5,98 \times 106$ & $0,0390^{*}$ \\
Fibrocartílago & $1,79 \times 107 \pm 2,77 \times 106$ & $1,69 \times 107 \pm 4,77 \times 106$ & 0,6493 \\
Tejido óseo trabecular & $8,10 \times 107 \pm 5,79 \times 106$ & $6,44 \times 107 \pm 1,66 \times 107$ & $0,0433^{*}$ \\
Tejido óseo compacto & $8,37 \times 107 \pm 1,22 \times 107$ & $1,17 \times 108 \pm 2,64 \times 107$ & $0,0167 *$ \\
*Diferencias significativas & & & \\
\hline
\end{tabular}

Tabla V. Análisis de correlación entre variables peso y volumen respecto a la proporción de área de los tejidos que constituyen el hueso sesamoídeo distal.

\begin{tabular}{lcc}
\hline & No Afectadas & Síndrome Navicular \\
& $\boldsymbol{r}$ & $\boldsymbol{r}$ \\
\hline Peso vs. Tejido óseo compacto $\left(\mu \mathrm{m}^{2}\right)$ & 0,8596 & 0,9682 \\
Peso vs. Tejido óseo trabecular $\left(\mu \mathrm{m}^{2}\right)$ & $-0,5831$ & 0,1819 \\
Peso vs. Fibrocartílago $\left(\mu \mathrm{m}^{2}\right)$ & $-0,2864$ & 0,8487 \\
Peso vs. Cartílago hialino $\left(\mu \mathrm{m}^{2}\right)$ & 0,2789 & 0,9116 \\
Volumen vs. Tejido óseo compacto $\left(\mu \mathrm{m}^{2}\right)$ & 0,7076 & 0,9544 \\
Volumen vs. Tejido óseo trabecular $\left(\mu \mathrm{m}^{2}\right)$ & $-0,7816$ & 0,2795 \\
Volumen vs. Fibrocartílago $\left(\mu \mathrm{m}^{2}\right)$ & $-0,5595$ & 0,8778 \\
Volumen vs. Cartílago hialino $\left(\mu \mathrm{m}^{2}\right)$ & 0,2095 & 0,8850 \\
\hline
\end{tabular}

Tabla VI. Parámetros estereológicos medidos en huesos sesamoídeo distal en equinos.

\begin{tabular}{|c|c|c|c|}
\hline \multirow{2}{*}{ 'ariable } & \multicolumn{2}{|c|}{ Promedio \pm DE } & \multirow{2}{*}{$p$} \\
\hline & No Afectados & Sínd rome Navicular & \\
\hline $\begin{array}{l}\mathrm{I}_{\mathrm{A}} \text { osteocitos - tejido óseo } \\
\text { abecular }\left(\mathrm{mm}^{2}\right) \text {. }\end{array}$ & $233,4 \pm 84,22$ & $258,6 \pm 60,65$ & 0,452 \\
\hline $\begin{array}{l}\mathrm{I}_{\mathrm{A}} \text { osteocitos - tejido óseo } \\
\text { ompacto }\left(\mathrm{mm}^{2}\right) \text {. }\end{array}$ & $329,1 \pm 109,1$ & $238,5 \pm 63,48$ & 0,028 \\
\hline $\begin{array}{l}{ }_{\mathrm{v}} \text { osteocitos - tejido óseo } \\
\text { abecular }\left(\mathrm{mm}^{0}\right) \text {. }\end{array}$ & $0,0340 \pm 0,02$ & $0,0620 \pm 0,0154$ & 0,003 \\
\hline $\begin{array}{l}{ }_{\mathrm{V}} \mathrm{v} \text { osteocitos - tejido óseo } \\
\text { ompacto }\left(\mathrm{mm}^{0}\right) \text {. }\end{array}$ & $0,0320 \pm 0,01$ & $0,0280 \pm 0,0078$ & 0,530 \\
\hline $\begin{array}{l}\mathrm{v} \text { condrocitos - cartílago } \\
\text { ialino }\left(\mu \mathrm{m}^{2} / \mu \mathrm{m}^{3}\right)\end{array}$ & $0,0165 \pm 0,008$ & $0,0128 \pm 0,007$ & 0,466 \\
\hline 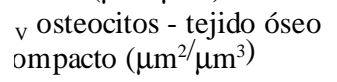 & $0,0157 \pm 0,007$ & $0,0168 \pm 0,009$ & 0,802 \\
\hline $\begin{array}{l}\text { v osteocitos - tejido óseo } \\
\text { abecular }\left(\mu \mathrm{m}^{2 /} \mu \mathrm{m}^{3}\right)\end{array}$ & $0,0106 \pm 0,01$ & $0,0159 \pm 0,009$ & 0,273 \\
\hline
\end{tabular}

*Diferencias significativas
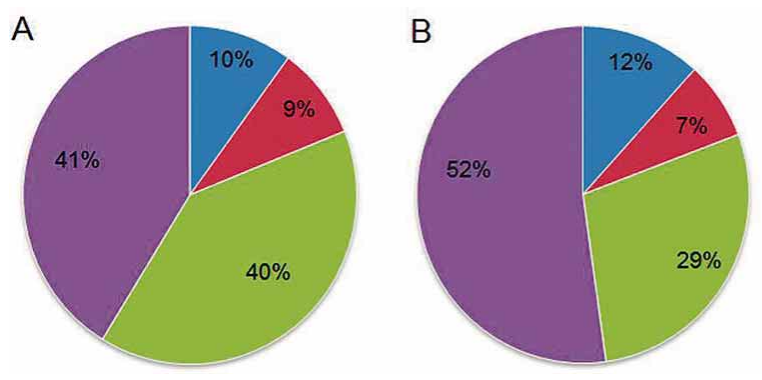

- Cartilago Hialino

- Fibrocartílago

- Tejido óseo

trabecular

- Tejido óseo

compacto

Fig. 3. Composicion del hueso sesamoídeo distal en equino en grupos (A) No Afectadas y (B) Síndrome Navicular. 
el área de cartílago hialino, de tejido óseo compacto y trabecular, y al contrario, no detectó diferencias entre grupos en el área de fibrocartílago. La Tabla $\mathrm{V}$ muestra el análisis de correlación entre las variables macroscópicas y el área de los tejidos que componen el hueso sesamoídeo distal. El análisis estadístico detectó una correlación positiva entre las variables peso y volumen respecto al área de tejido óseo compacto en ambos grupos, además, se observó una correlación negativa entre el peso y volumen respecto al área de tejido óseo trabecular solo en el grupo No Afectadas. Por último, detectó en el grupo Síndrome Navicular, una correlación positiva entre las variables peso y volumen respecto al área de cartílago hialino y fibrocartílago. La Tabla VI muestra los parámetros estereológicos medidos en tejido óseo compacto y trabecular. El análisis estadístico detectó diferencias significativas entre grupos en el número de perfiles osteocitarios $/ \mathrm{mm}^{2}$ en tejido óseo compacto y en el volumen de osteocitos en el tejido óseo trabecular.

\section{DISCUSIÓN}

El procedimiento de obtención de imágenes radiográficas y los criterios de evaluación para la formación de grupos utilizados en el estudio han sido realizados según lo descrito por Kinast y Floyd \& Mansmann. Komosa et al. (2013) afirman que la alta incidencia de la observación de hallazgos radiográficos tales como pérdida de definición córtico medular, asociados a una esclerosis y osteólisis de tejido óseo compacto en la cara flexora y remodelación de los márgenes proximal y distal del HSD, se relacionan con la presentación clínica del SN en animales vivos. Otros autores (Bentley et al., 2007; Waguespack \& Hanson, 2010; Dyson et al.) afirman que, frente a las posibles etiopatogenias del SN, se generan cambios morfológicos en el HSD que se evidencian en signos de dolor y/o hallazgos imagenológicos, observándose alteraciones no sólo en el HSD sino también en estructuras adyacentes. Blunden et al. (2007) describieron una avanzada remodelación ósea, demostrando la existencia de procesos patológicos que lo afectarían, como respuesta a los apremios mecánicos debidos a altas exigencias (Kinast), todos relacionados al SN. Por lo tanto, creemos que los criterios diagnósticos en las manos y la conformación de grupos son representativos de equinos con SN.

El estudio morfométrico demostró que no hay diferencias significativas en cuanto a peso, volumen y medidas lineales. Nuestros resultados coinciden con lo reportado por Murray et al. (2006) quienes utilizaron la resonancia nuclear magnética para evaluar el HSD, y en cuyo estudio no se observó diferencias en la morfología macroscópica entre equinos con y sin SN. Sin embargo, no concuerdan con lo reportado por Waguespack \& Hanson, Dyson et al., y Komosa et al. quienes describen variaciones morfológicas durante el desarrollo y curso del $\mathrm{SN}$. Creemos que la ausencia de cambios morfológicos significativos observados en nuestro estudio se debe a una falla en la adaptación funcional del HSD. Es conocido que en las superficies donde se ejercen presiones ocurre resorción ósea y el hueso busca espacio para depositar nuevo tejido óseo (Junqueira \& Carneiro, 1996), por lo tanto, debería aumentar su tamaño excéntricamente para soportar las demandas biomecánicas (Dellman \& Eurell, 1998), sin embargo, al encontrarse inmerso entre estructuras rígidas, éstas limitarían su fortalecimiento y crecimiento, mas aún cuando se encuentran incluídas en la úngula, que en animales con estructuras córneas descuidadas determinarían una mayor presión sobre el HSD limitando el desarrollo compensatorio (Bentley et al.). La restricción ejercida por la herradura, especialmente en la región palmar, impide que se ejecute la absorción de choque normal de la úngula. Otro factor importante que determina esta falla adaptativa es el inicio temprano en actividades de esfuerzo. Es importante señalar que el desarrollo óseo del equino concluye a los 5,5 años. Komosa et al. mencionan que un desequilibrio en la interacción entre el modelado óseo (propio del crecimiento y desarrollo) con el remodelado óseo (propio de la adaptación funcional) causado por una situación de estrés y a las características anatómicas del HSD, resultan en un fracaso adaptativo. Bentley et al., explican esta última condición señalando que la falta de adaptación funcional para proteger al HSD del daño es común, afirmando que las cargas cíclicas aplicadas durante la actividad atlética, el uso de herraduras o cualquier factor que repercuta negativamente en la "propiedades visco-elásticas" probablemente hacen aumentar la deformación ósea.

El estudio planimétrico demostró diferencias en la proporción de los componentes tisulares, que indicarían que el SN produce cambios a nivel mesoscópico en el HSD. Se detectaron diferencias entre grupos en el área de tejido óseo compacto, trabecular y cartílago hialino. Nuestros resultados coincidieron con lo reportado por Gabriel et al. (1998), quienes demostraron que los HSD de equinos atléticos presentaron grandes cantidades de tejido óseo compacto y una menor cantidad de tejido óseo trabecular, comparados con equinos sedentarios. Se ha demostrado en una serie de estudios que el ejercicio aumenta la masa media, el grosor y la fuerza estructural del HSD (Buckingham \& Jeffcott, 1990) este aumento en la tensión mecánica estimula el crecimiento y modelado, sin embargo deprime la resorción ósea. Nuestro estudio demostró que aquellos 
huesos de equinos con SN presentaron una mayor área de tejido óseo compacto y una reducida área de tejido óseo trabecular. Esto, lo atribuimos, a que las manos diagnosticadas con SN eran de animales sometidos a fuertes, constantes y excesivas presiones sobre el HSD. Las características morfológicas externas medibles como peso y volumen se correlacionan positivamente con el área del tejido óseo compacto en ambos grupos, siendo esta condición consecuente con los cambios mesoscópicos adaptativos reportados por Gabriel et al. Creemos que la alta densidad de osteocitos en el tejido óseo compacto determina una mayor área y peso, aumentando además, la matriz extracelular. Además, la variable volumen demostró una correlación positiva con el área de cartílago hialino y fibrocartílago en manos con SN, siendo consecuencia de la adaptación a las constantes presiones ejercidas sobre el HSD.

El estudio estereológico demostró diferencias en la cantidad y volumen de osteocitos en el tejido óseo compacto y trabecular, respectivamente. Los resultados indicarían que el SN produce cambios celulares cuantitativos en el HSD. Coincidimos con lo reportado por Bentley et al., quienes realizaron un análisis de la adaptación funcional del HSD en equinos con SN y reportaron una baja densidad osteocitaria (34-248 osteocitos $/ \mathrm{mm}^{2}$ ). El tejido óseo compacto del HSD debe soportar la mayor cantidad de cargas y presiones (Kinast; Bentley et al.; Floyd \& Mansmann; Waguespack \& Hanson; Dyson et al.; Komosa et al.), así los equinos con una correcta conformación ósea, y manejo adecuado de la úngula, presentan una distribución y resistencia homogénea de las cargas aplicadas sobre el HSD (Stashak). Se ha descrito que las fuerzas que no superan un cierto umbral mantienen la viabilidad de los osteocitos (Aguirre et al., 2006), sin embargo, al existir un estado patológico, causado por factores de estrés biomecánico, manejos inadecuados y sobre exigencia, descritos como causas probables de $\mathrm{SN}$, disminuyen la cantidad y volumen de osteocitos en el tejido óseo compacto determinados por la muerte osteocitaria, además, el desarrollo de microfracturas determinan la actividad osteoclástica en estos sitios e inician procesos de resorción ósea potenciado por un aporte vascular disminuido (Komosa et al.). El tejido óseo trabecular proporciona un apoyo eficaz para la transferencia de cargas de compresión, y aumenta la flexibilidad y amortiguación de un hueso bajo tensión (Gabriel et al.). En el HSD, las trabéculas están orientadas en una dirección próximo-distal dorsopalmar, por lo tanto, adaptadas para resistir la compresión ejercida por el tendón del músculo flexor digital profundo y por la falange media que transmite una parte del peso corporal al HSD durante la carga (Gabriel et al.). Esta mejor capacidad adaptativa determina la viabilidad y evita la muerte osteocitaria. Creemos que el aumento del volumen en el tejido óseo trabecular en equinos con SN es una consecuencia adaptativa y una señal de osteocitos viables. El tejido óseo trabecular no está directamente expuesto a la presión y es el tejido óseo compacto el que recibe y atenúa las cargas, provocando en él, mayor daño (Bentley et al.; Waguespack \& Hanson; Dyson et al.). Por esto, la integridad del tejido óseo trabecular se mantiene, favoreciendo la adecuada comunicación intersticial (lacuno canalicular) (Dellman \& Eurell).

En conclusión, el SN no modifica la morfología macroscópica del HSD en manos equinas, sin embargo modifica la proporción del área de los tejidos que lo constituyen, además, disminuye la cantidad de osteocitos en el tejido óseo compacto, aumentando su volumen en el tejido óseo trabecular. Por lo tanto, a pesar de existir un proceso patológico e inflamatorio en la región del HSD que induce cambios mesoscópicos y microscópicos atribuibles a SN, éstos no logran modificar sus características macroscópicas.

SAlinAS, P.; FIGUEROA, S.; CARRASCO, C. \& BAÑADOS, R. Morphometry, planimetry and stereology in the distal sesamoid bone in equine foot with and without Navicular Syndrome. Int. J. Morphol., 32(1):357-363, 2014.

SUMMARY: Navicular Syndrome (NS) is a chronic, degenerative and progressive bilateral claudication pathology, compromising the distal sesamoid bone (DSB), the podotrochlear bursa, ligaments and adjacent surfaces of the tendon of the deep digital flexor muscle of equine foot. The hypothesis that morphological characteristics of HSB in hand of horses vary in those with SN was evaluated in this study. A study was carried out to determine the morphological changes in the DSB in 22 left foot of horses with NS. We realized morphometrical, planimetrical and stereological studies in two groups of horses feet: Not Affected ( $n=11)$ and Navicular Syndrome $(n=11)$. The following variables were quantified: weight, volume, linear measurements, area and stereological parameters: densities of number $\left(\mathrm{N}_{\mathrm{A}}\right)$, volume $\left(\mathrm{V}_{\mathrm{v}}\right)$ and surface $\left(\mathrm{S}_{\mathrm{v}}\right)$. Results indicate that although there is a pathological and inflammatory process in the region of DSB which induces mesoscopic and microscopic changes attributable to SN, their gross morphological features were not modified.

KEY WORDS: Equine; Navicular syndrome; Distal sesamoid bone; Morphometry; Planimetry; Stereology. 


\section{REFERENCIAS BIBLIOGRÁFICAS}

Aguirre, J. I.; Plotkin, L. I.; Stewart, S. A.; Weinstein, R. S.; Parfitt, A. M.; Manolagas, S. C. \& Bellido, T. Osteocyte apoptosis is induced by weightlessness in mice and precedes osteoclast recruitment and bone loss. J. Bone. Miner. Res., 21(4):605-15, 2006.

Bentley, V.; Sample, S.; Livesey, M.; Scollay, M.; Radtke, C.; Frank, J.; Kalscher, J. \& Muir, P. Morphologic changes associated with functional adaptation of the navicular bone of horses. J. Anat., 211(5):662-72, 2007.

Blunden, A.; Dyson, S.; Murray, R. \& Schramme, M. Histopathology in horses with chronic palmar foot pain and age-matched controls. Part 1: Navicular bone and related structures. Equine Vet. J., 38(1):15-22, 2007.

Bowker, R.; Atkinson, P.; Atkinson, T. \& Haut, R. Effect of contact stress in bone of the distal interphalangeal joint on microscopic changes in articular cartilage and ligaments. Am. J. Vet. Res., 62(3):414-24, 2001.

Buckingham, S. H. W. \& Jeffcott, L. B. The skeletal effects of a long term submaximal exercise programme on standardbred yearlings. In: Persson, S. G. B.; Lindholm, A. \& Jeffcott, L. B. Equine Exercise Physiology 3. Davis, ICEEP Publications. 1991. pp.411-8.

Dellmann, H. \& Eurell, J. Textbook of Veterinary Histology. 5th ed. Philadelphia, Lippincott Williams \& Wilkins, 1998.

Dorph-Petersen, K. A.; Nyengaard, J. R. \& Gundersen, H. J. G. Tissue shrinkage and unbiased stereological estimation of particle number and size. J. Microsc., 204(3):232-46, 2001.

Dyson, S.; Murray, R.; Schramme, M. \& Blunden, T. Current Concepts of Navicular Disease. Equine Vet. Educ. 23(1):2739, 2011.

Federative International Committee on Anatomical Terminology. Terminología Histológica. International Terms for Human Cytology and Histology. Philadelphia, Wolters Kluwer/Lippincott Williams \& Wilkins, 2008. pp.19-23, 38-40.

Floyd, A. E. \& Mansmann, R. A. Podiatría Equina. Bueno Aires, Intermédica. 2009. pp.4-9, 30-1.

Gabriel, A.; Jolly, S.; Detilleux, J.; Dessy-Doize, C.; Collin, A. \& Reginster, B. Morphometric study of the equine navicular bone: variations with breeds and types of horse and influence of exercise. J. Anat., 193(Pt. 4):535-49, 1998.

International Committee on Veterinary Gross Anatomical Nomenclature. Nomina Anatómica Veterinaria. $5^{\text {th }}$ ed. New York, Editorial Committee, 2005.
Junqueira, L. C. \& Carneiro J. Histología Básica Texto y Atlas. $4^{\text {o }}$ ed. Madrid, Masson. 1996. pp.137-40, 150-3.

Kinast, C. Caracterización del hueso navicular del caballo chileno mediante las técnicas de examen radiológico "vía coronaria alta" y la "vista tangencial flexora". Tesis pregrado, Universidad Austral de Chile, Facultad de Ciencias Veterinarias, Valdivia, Chile, 2011.

Komosa, M.; Purzyc, H. \& Frackowiak, H. Changes in navicular bone (os sesamoideum distale) shape in horse as a result of pathological alterations. Folia Biol. (Krakow), 61(1-2):1$10,2013$.

Mandarim-de-Lacerda, C. A. Stereological tools in biomedical research. An. Acad. Bras. Cienc., 75(4):469-86, 2003.

Murray, R.; Schramme, M.; Dyson, S.; Branch, M. \& Blunden, T. Magnetic resonance imaging characteristic of the foot in horses with palmar foot pain and control horses. Vet. Radiol. Ultrasound, 47(1):1-16, 2006.

Stashak, T. Adams' Lameness in horses. $4^{\text {th }}$ Ed. Philadelphia, Lea \& Febiger. 2004. pp.113-17, 706-22.

Tschanz, S. A.; Burri, P. H. \& Weibel, E. R. A simple tool for stereological assessment of digital images: the STEPanizer. J. Microsc., 243(1):47-59, 2011.

Voute, L. What can radiology tell us about palmar foot pain? Proceedings of the $47^{\text {th }}$. Brit. Eq. Vet. Assoc. Cong., 2008. pp.30-2.

Waguespack, R. \& Hanson, R. Navicular Syndrome in equine patients: anatomy, causes, and diagnosis. Compend. Contin. Educ. Pract. Vet., 32(12):E7, 2010.

Wilson, A. M.; Mcguigan, M. P.; Fouracre, L. \& Macmahon, $\mathrm{L}$. The force and contact stress on the navicular bone during trot locomotion in sound horses and horses with navicular disease. Equine Vet. J., 33(2):159-65, 2001.

Dirección para correspondencia

Paulo C. Salinas Pérez

Escuela de Medicina Veterinaria

Universidad Santo Tomás

Rodríguez 060, Temuco

CHILE

Email: psalinas@santotomas.cl
Recibido : 03-12-2013

Aceptado: 22-01-2014 